\title{
LES HYALOMMA (ACARIENS, IXODIDAE) DE FRANCE
}

\author{
Par P.-C. MOREL
}

Depuis une dizaine d'années, divers auteurs ont signalé la présence dans le Sud de la France de tiques du genre Hyalomma. Quoique un travail d'ensemble sur la répartition des espèces n'ait pas été entrepris, il me semble utile de réunir ici les renseignements que nous fournit la littérature à ce sujet, ainsi que les résultats de l'examen de plusieurs collections. Une connaissance exacte de cette répartition serait d'autant plus importante que certains Hyalomma sont reconnus vecteurs de rickettsioses et de theilerioses.

\section{HYALOMMA MARGINATUM Koch, 1844.}

La seule référence sûre concernant le continent est celle de Hoogstraal (1956, p. 469) pour le Mont-Ėstérel (Var). L'espèce semble abondante en Corse, peut-être introduite anciennement par du bétail de Sardaigne ou de la Péninsule italique ; Delpy (1946) la signale de Corse sur Corvus corone (nymphes recueillies par Brumpt et mises en élevage) ; dans la collection Brumpt (Faculté de Médecine de Paris) se trouvent les lots suivants : $\delta^{7} \sigma^{7}$ de Casabranda (4-VII-25) sur mulet, $2 q$ de Furlani (VII-25) sur bœuf,

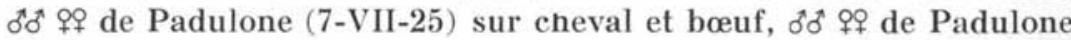
sur hérisson (probablement recueillis sur cet hôte à l'état nymphal) ; dans la collection Neumann à Toulouse existe $1 q$ de Bastia $\left(n^{\circ}\right.$ 934, Dumestre legit 1893) ; R.-Ph. Dollfus possède 3 ơ de Corse pris sur sanglier (Houdemer legit 1951). Dans la collection du Museum (laboratoire de M. Marc André), j'ai vu une $q$ d'Ajaccio (Dehaut legit 1905).

Je ne sais pas ce que représente le $H$. marginatum de Enigk (1947), de Urt (B.-P.), sur cheval (1-VI-44) ; sa carte de répartition de $H$. savignyi (1954) [synonyme de marginatum selon Delpy (1949) ou de excavatum selon Feldman-Muhsam (1954)] correspond plutôt à la répartition de $H$. excavatum, notamment en ce qui concerne la France.

Ann. de Parasitologie, T. XXXIII, N ${ }^{\circ} 4,1959$. 
De même, le savignyi sensu Delpy de Lamontellerie (1954) me semble nécessiter confirmation. S'il n'est pas impossible a priori que marginatum soit présent dans le Sud-Ouest, la photographie que donne l'auteur p. 49 fait plutôt penser à $H$. excavatum par la parma (feston postéro-médian) décolorée, qui est un caractère très suffisant pour distinguer les deux espèces, quand cette parma est bien distincte (cf. infra).

H. marginatum est présent en Espagne, Portugal et Italie.

HYALOMMA EXCAVATUM Koch, 1844.

Cette espèce est signalée par les auteurs suivants : Brumpt \& Chabaud (1947 : Desportes legit) : Camargue ; Théodoridès (1951 \& 1953) : Banyuls (27-XI-50), La Madeloc (IX-52, Remmert legit) ; Colas-Belcour \& Rageau (1951): Banyuls (Théodoridès legit). Le savignyi de Lamontellerie (1954) (= excavatum?) a été récolté dans les départements des Landes, Gironde et Charente-Maritime, sur bœuf, cheval et homme.

J'ai examiné les exemplaires suivants : $\delta \delta^{\circ}$ 우 de Camargue (Coll. Laboratoire de Parasitologie Alfort: Sinodinos legit) (VI-49) : bovins ; $\delta^{*}+\%$, Salis-de-Badon (Camargue) (VII-54) : sur les herbes (coll. personnelle) ; 86 \$क, Le Sambuc (Camargue) (5-VII-54) : sanglier (coll. pers.) ; 1 б, La Massane (Banyuls) (IV-53) : sur herbes (coll. pers.) ; 4 б, 2 , Roquebrune-sur-Argens (Var) : terrier de lapins (coll. Inst. Pasteur Paris : Lègue legit, 1948).

Il y a grande chance que l'espèce soit très anciennement établie en France, importée par les oiseaux migrateurs, mais qu'elle n'ait jamais été mise en évidence du fait de la rareté des collecteurs.

Je dois signaler que les exemplaires que j'ai vus de Camargue, du Var et des Pyrénées-Orientales correspondent à la forme lusitanicum (enduit blanchâtre et parma non distincte, car très chitinisée, comme les autres festons), qui est peut-être pour le moins une forme géographique distincte de excavatum, avec lequel Delpy (1949) l'avait mis en synonymie. Tendeiro (1955) le réhabilite au rang d'espèce. Il a peut-être raison, mais ceci demande confirmation par des élevages.

Le fait curieux est alors la présence possible de la forme typique excavatum dans le Sud-Ouest (si la rectification des Hyalomma de Lamontellerie s'avère exacte par la suite). 


\section{Conclusions}

a) H. marginatum est sùrement présent $€$ n Corse et dans l'Estére!.

b) H. excavatum est présent dans le Sud méditerranéen (forme lusitanicum).

c) Un Hyalomma sp. (probablement $H$. excavatum forme typique) est présent dans le Sud-Ouest.

d) Devant le caractère incomplet de ces renseignements, les parasitologues sont invités à récolter des tiques; nous nous ferons un plaisir de les déterminer et de centraliser les résultats.

\section{BIBLIOGRAPHIE}

1. Brumpt (E.) et Chabaud (A.) (1947). - L'infestation par des Ixodinés provoque-t-elle une immunité chez l'hôte? Note préliminaire. Ann. Parasit., XXII, (5-6) : 348-356.

2. Colas-Belcour (J.) et Rageau (J.) (1951). - Tiques de Tunisie. Arch. Inst. Pasteur Maroc, IV, (4) : 360-367.

3. Delpy (L. P.) (1946). - Révision par des voies expérimentales du genre Hyalomma C. L. Koch, 1844, Note préliminaire. Ann. Parasit., XXI, (5-6) : 267-293.

4. - (1949). - Idem, $2^{\circ}$ partie. Ibidem, XXIV, (1-2) : 97-109.

5. - (1949). - Essai critique de synonymie du genre Hyalomma C. L. Koch, 1844, depuis Linné, 1758 ; Ibidem, XXIV, (5-6) : 464-494.

6. Exıgk (K.) (1947). - Zur Kenntnis der Zecken von Süd- und Osteuropa. Mh. vet. Med., II, (5) : 75-81.

7. Exick (K.) (1954). - Zur Biologie des Zecken. Deut. Entomologentag in Hambourg, Jena (G. Fischer), 96-102.

8. Hoogstrade (H.) (1956). - African Ixodoidea. I. Ticks of the Sudan. Research Report of the U.S. Naval Medical Research Unit, $\mathrm{n}^{\circ} 3$, NM 005 050.29.07, Gov. Print. Office, 1956-0-390, 800, $1101 \mathrm{pp.}$

9. Lamontellerie (M.) (1954). - Les Ixodoüdes du Sud-Ouest de la France. Espèces rencontrées, agressivité, rôle pathogène. Bordeaux (E. Drouillard), $145 \mathrm{pp}$.

10. Tendeiro (J.) (1955). - Sobre alguns Ixodideos dos généros Hyalomma C. L. Koch, 1844 e A ponomma Neumann, 1899. Bol. cult. Guiné port., X, (39) : 319-461.

11. ThÉononidès (J.) (1951). - Capture de Hyalomma excavatum C. L. Koch dans la région de Banyuls. Vie et Milieu, II, (1) : 155-156.

12. - (1953). -- Première contribution à l'étude des ectoparasites des Vertébrés des Pyrénées Orientales. Vie et Milieu, IV, (4) : 753-756. 


\section{NOTE ADDITIONNELLE}

Dans une publication dont j'ai reçu copie dactylographiée (sous presse), Hoogstraal et Kaiser établissent, d'une manière qu'on ose espérer définitive, le statut systématique de certaines espèces et sous-espèces de Hyalomma du groupe excavatum sensu Delpy. Me fondant sur cette autorité, je rectifie comme suit la terminologie utilisée dans la note sur les Hyalomma de France :

a) H. lusitanicum Koch, 1844 est une bonne espèce (présente en Italie, France et Péninsule ibérique).

b) Ce que je désigne par $H$. excavatum doit ètre nommé $H$. anatolicum anatolicum Koch, 1844 (présent en Méditerranee orientale et sur la côte du Maghreb ; présence en France problématique). H. anatolicum excavatum Koch, 1844 représente une forme de la précédente, plus rare et de biotope plus désertique, ayant à peu près la même aire de répartition.

c) Le groupe excavatum sensu Delpy doit être nommé à l'avenir groupe anatolicum.

\section{Bibliographie}

Hoogstrade. (H.) et Kaiser (M. N.) (1958), - Observations on egyptian Hyalomma ticks (Ixodoidea, Ixodidw). 5. Biological notes and differences in identity of $H$. anatolicum and its subspecies anatolicum Koch and excavatum Koch among russian and other workers. Identity of $\mathrm{H}$. Iusitanicum. Ann. entom. Soc. Amer. (sous presse). 